\title{
Is the Invariance with Respect to Powers of a t-norm a Restrictive Property on Fuzzy Implication Functions? The Case of Strict t-norms
}

\author{
Raquel Fernandez-Peralta ${ }^{1,2}(\mathbb{D})$, Sebastia Massanet ${ }^{1,2(\bowtie)} \mathbb{D}^{\mathbb{D}}$, and Arnau Mir $^{1,2}(\mathbb{D}$ \\ 1 Soft Computing, Image Processing and Aggregation (SCOPIA) Research Group, \\ Department Mathematics and Computer Science, University of the Balearic Islands, \\ 07122 Palma, Spain \\ $\{$ r.fernandez, s.massanet, arnau.mir\}@uib.es \\ 2 Balearic Islands Health Research Institute (IdISBa), 07010 Palma, Spain
}

\begin{abstract}
The invariance with respect to powers of a t-norm has emerged as an important property for fuzzy implication functions in approximate reasoning. Recently, those fuzzy implication functions satisfying this property where fully characterized leading to seemingly new families of these operators. In this paper, the additional properties of the family of fuzzy implication functions which are invariant with respect to powers of a strict t-norm are analyzed. In particular, properties such as the exchange principle, the law of importation with respect to a t-norm or the left neutrality principle, among others, can be fulfilled by some members of this family. This study allows to characterize the intersection of these operators with the most important families of fuzzy implication functions.
\end{abstract}

Keywords: Fuzzy implication function - Invariance $\cdot$ Powers of t-norms $\cdot$ Exchange principle

\section{Introduction}

In the last decades, dozens of families of fuzzy implication functions have been proposed in the literature (see $[1,2,5]$ and references therein). Although some of these families have boosted some applications in which fuzzy implication functions play a key role, other families struggle to stand out since they do not satisfy any differentiating additional property with respect to the rest. Moreover, this vast number of families is starting to cause some major problems in the research of the field [6]. Therefore, it is necessary to analyze in depth those families of fuzzy implication functions which provide uncommon but useful properties and to analyze their relationship and intersections with other well-known families. This will allow the community to disclose more about the structure of these operators and to open new potentially useful lines of research.

(C) Springer Nature Switzerland AG 2020

M.-J. Lesot et al. (Eds.): IPMU 2020, CCIS 1238, pp. 761-774, 2020.

https://doi.org/10.1007/978-3-030-50143-3_59 
In this direction, the invariance property with respect to powers of a continuous t-norm was proposed in [8] as an additional property of fuzzy implication functions with applications in approximate reasoning. As it is stated in [8], the fulfillment of this property ensures that the following fuzzy propositions from the classical example given in [10]:

If the tomato is red, then it is ripe.

If the tomato is very red, then it is very ripe.

If the tomato is little red, then it is little ripe.

have the same truth value whenever the linguistic modifiers "very" and "little" are modeled using powers of continuous t-norms. This additional property is not satisfied in general by the most usual families of fuzzy implication functions. Therefore, in [8], the so-called $T$-power based implications are introduced as a family of fuzzy implication functions satisfying the invariance for many t-norms (see the corrigendum [7] also). Later, in [9], the complete characterization of all fuzzy implication functions satisfying the invariance property with respect to powers of a continuous t-norm is achieved. Indeed, the characterization depends on the type of continuous t-norm and provides the expression of the family of fuzzy implication functions fulfilling the property. However, in [9], only this property is studied and up to now, it is unknown which other additional properties can be satisfied by the members of these families of invariant implications. Thus, the goal of this paper is to study which well-known additional properties these fuzzy implication functions satisfy and under which conditions. As a first approach to this problem, this paper deals with the family of fuzzy implication functions which are invariant with respect to powers of a strict t-norm. This study will encourage the use of fuzzy implication functions in approximate reasoning where other additional properties may be required in addition to the invariance property. Moreover, as a straightforward consequence, the study of the additional properties of this family allows to determine the intersection of this family with some of the most important families of fuzzy implication functions, namely $(S, N), R, Q L$ and Yager's $f$ and $g$ generated implications (see $[1])$.

The paper is organized as follows. In the next section we recall some basic definitions and properties on fuzzy implication functions. In Sect. 3, the family of fuzzy implication functions which are invariant with respect to powers of strict t-norms is recalled and its definition is revisited. Then, in Sect. 4, the additional properties of the family are deeply analyzed and the conditions under which this family fulfills them are determined. After that, in Sect.5, the intersections of this family with some well-known families is derived from the study carried out in the previous section. The paper ends with some conclusions and future work.

\section{Preliminaries}

To make this work self-contained, we recall here some of the concepts and results which will be used throughout the paper. Although we will suppose the reader 
is familiar with basic results on t-norms (see [4,12] for more details), we recall the definition of a strict t-norm and the expression of its powers.

Definition 1 ([4]). A function $T:[0,1]^{2} \rightarrow[0,1]$ is called a strict t-norm if there exists a continuous, strictly decreasing function $t:[0,1] \rightarrow[0,+\infty]$ with $t(0)=+\infty$ and $t(1)=0$, which is uniquely determined up to a positive multiplicative constant, such that $T$ is given by

$$
T(x, y)=t^{-1}(t(x)+t(y))
$$

for all $x, y \in[0,1]$.

Powers of a t-norm $T$, which are defined in detail in [12] and will be denoted by $x_{T}^{(r)}$ with $x \in[0,1]$ and $r \in[0,+\infty]$, can be expressed for strict t-norms $T$ in terms of an additive generator of the t-norm.

Proposition 1 ([12]). Let $T$ be a strict $t$-norm with additive generator $t$. Then

$$
x_{T}^{(r)}=t^{-1}(r t(x)) \text { for all } x \in[0,1] \text { and } r \in[0,+\infty]
$$

with the convention that $+\infty \cdot 0=0$.

We start now with the definition of a fuzzy implication function.

Definition 2 ([1,3]). A binary operator $I:[0,1]^{2} \rightarrow[0,1]$ is said to be a fuzzy implication function if it satisfies:

(I1) $I(x, z) \geq I(y, z)$ when $x \leq y$, for all $z \in[0,1]$.

(I2) $I(x, y) \leq I(x, z)$ when $y \leq z$, for all $x \in[0,1]$.

(I3) $I(0,0)=I(1,1)=1$ and $I(1,0)=0$.

From the definition, it can be easily derived that $I(0, x)=1$ and $I(x, 1)=1$ for all $x \in[0,1]$. On the other hand, the symmetrical values $I(x, 0)$ and $I(1, x)$ are not predetermined from the definition.

Along the history of fuzzy implication functions, additional properties of these functions have been postulated (see $[1,3,11]$ for more details). Among the most important and those that are relevant for this work we stand out the following ones:

- The identity principle

$$
I(x, x)=1, \quad x \in[0,1] .
$$

- The ordering property

$$
I(x, y)=1 \Leftrightarrow x \leq y, \quad x, y \in[0,1] .
$$

- The exchange principle

$$
I(x, I(y, z))=I(y, I(x, z)), \quad x, y, z \in[0,1] .
$$


- The law of importation with respect to a t-norm $T$

$$
I(T(x, y), z)=I(x, I(y, z)), \quad x, y, z \in[0,1] .
$$

- The left neutrality principle

$$
I(1, y)=y, \quad y \in[0,1] .
$$

- The iterative boolean law

$$
I(x, y)=I(x, I(x, y)), \quad x, y \in[0,1] .
$$

In addition to the previous additional properties, the invariance property with respect to t-norms was recently proposed in [8] in order to deal with the classical problem of the tomato recalled in the introduction.

Definition 3 ([8]). Let I be a fuzzy implication function and $T$ a continuous t-norm. It is said that $I$ is invariant with respect to $T$-powers, or simply that it is $T$-power invariant when

$$
I(x, y)=I\left(x_{T}^{(r)}, y_{T}^{(r)}\right),
$$

holds for all real number $r>0$ and for all $x, y \in[0,1]$ such that $x_{T}^{(r)}, y_{T}^{(r)} \neq 0,1$.

\section{$3 \quad$ Strict $\boldsymbol{T}$-power Invariant Implications}

In [9] all fuzzy implication functions which are invariant with respect to $T$-powers when $T$ is a strict t-norm were characterized in the following theorem.

Theorem 1 ([9, Theorem 8]). Let $T$ be a strict $t$-norm and $t$ an additive generator of T. A mapping $I:[0,1]^{2} \rightarrow[0,1]$ is a fuzzy implication function invariant with respect to $T$-powers if and only if there exists an increasing mapping $\varphi:[0,+\infty] \rightarrow[0,1]$ with $\varphi(0)=0, \varphi(+\infty)=1$ and such that $I$ is given by

$$
I(x, y)=\varphi\left(\frac{t(x)}{t(y)}\right), \quad \text { for all }(x, y) \in[0,1]^{2} \backslash\{(x, 0),(1, y) \mid 0<x, y<1\},
$$

with the convention that $\frac{0}{0}=\frac{+\infty}{+\infty}=+\infty$, and such that the remaining values $I(x, 0)$ and $I(1, y)$ preserve the monotonicity conditions.

The aim of this paper is to study the family of fuzzy implication functions described in the theorem above. In order to do so, we first provide a concrete definition of such family and we establish the conditions which ensure that these functions are indeed fuzzy implication functions.

Let us consider $T$ a strict t-norm, $t$ an additive generator of $T$ and $I$ : $[0,1]^{2} \rightarrow[0,1]$ a fuzzy implication function invariant with respect to $T$-powers. 
From Theorem 1 we know that there exists an increasing mapping $\varphi:[0,+\infty] \rightarrow$ $[0,1]$ with $\varphi(0)=0, \varphi(+\infty)=1$ and such that $I$ is given by $(1)$.

First of all, notice that since the additive generator of a t-norm is unique up to a positive multiplicative constant, the definition of $I$ is independent from the considered additive generator of $T$. Let us define $f(x)=I(x, 0)$ for all $x \in(0,1)$ and $g(y)=I(1, y)$ for all $y \in(0,1)$. Now, in order for $I$ to be a fuzzy implication function, the functions $f$ and $g$ need to respect the monotonicity conditions (I1) and (I2). From (I2) we get that $g$ has to be an increasing function and, for a fixed $x \in(0,1)$ the following condition must hold

$$
f(x)=I(x, 0) \leq I(x, y)=\varphi\left(\frac{t(x)}{t(y)}\right), \quad \text { for all } y \in(0,1) .
$$

On the other hand, from (I1) we get that $f$ has to be a decreasing function and, for a fixed $y \in(0,1)$ the following condition must hold

$$
g(y)=I(1, y) \leq I(x, y)=\varphi\left(\frac{t(x)}{t(y)}\right), \quad \text { for all } x \in(0,1)
$$

Now, it is easy to prove that inequalities (2) and (3) hold if and only if

$$
\inf _{w \in(0,+\infty)} \varphi(w) \geq \max \left\{\sup _{y \in(0,1)} g(y), \sup _{x \in(0,1)} f(x)\right\} .
$$

Having said this, we provide the following definition of the family of fuzzy implication functions that are $T$-power invariant with respect to a strict t-norm.

Definition 4. Let $T$ be a strict $t$-norm and $t$ an additive generator of $T$. Let $f:(0,1) \rightarrow[0,1]$ be a decreasing function and $\varphi:[0,+\infty] \rightarrow[0,1], g:(0,1) \rightarrow$ $[0,1]$ increasing functions such that $\varphi(0)=0, \varphi(+\infty)=1$ and

$$
\inf _{w \in(0,+\infty)} \varphi(w) \geq \max \left\{\sup _{y \in(0,1)} g(y), \sup _{x \in(0,1)} f(x)\right\} .
$$

The function $I_{\varphi, f, g}^{T} ;[0,1]^{2} \rightarrow[0,1]$ defined by

$$
I_{\varphi, f, g}^{T}(x, y)= \begin{cases}f(x) & \text { if } x \in(0,1) \text { and } y=0 \\ g(y) & \text { if } x=1 \text { and } y \in(0,1) \\ \varphi\left(\frac{t(x)}{t(y)}\right) & \text { otherwise }\end{cases}
$$

with the understanding $\frac{0}{0}=\frac{+\infty}{+\infty}=+\infty$, is called a strict T-power invariant implication.

In Fig. 1 we can see the structure of fuzzy implication functions given by Expression (5). 


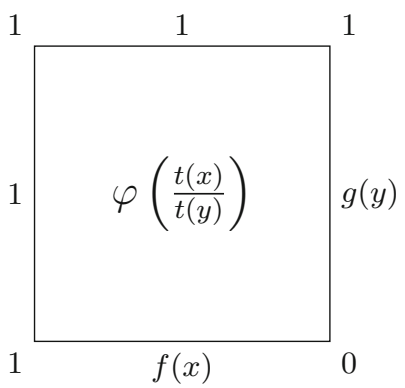

Fig. 1. Structure of the family of strict $T$-power invariant implications.

Notice that Condition (4) imposes that the function $\varphi$ is bounded below by any possible value of $f$ and $g$ (see Example 1 ). Although the structure of strict $T$ power invariant implications may seem flexible since it depends of three unknown functions, as a matter of fact, Condition (4) severely restricts the choices of functions $\varphi, f$ and $g$ for which $I_{\varphi, f, g}^{T}$ is a fuzzy implication function. Indeed, the following proposition studies the continuity of strict $T$-power implications and shows that certain desired properties of $I_{\varphi, f, g}^{T}$ lead to impose that $\varphi, f$ or $g$ are constant functions.

Proposition 2. Let $I_{\varphi, f, g}^{T}$ be a strict T-power invariant implication. The following statements hold:

(i) If $\varphi$ is continuous at $w=0$, then $f(x)=g(y)=0$ for all $x, y \in(0,1)$.

(ii) If $\lim _{x \rightarrow 0^{+}} f(x)=1$ or $\lim _{y \rightarrow 1^{-}} g(y)=1$, then $\varphi(w)=1$ for all $w \in(0,1)$.

(iii) $I_{\varphi, f, g}^{T}$ is continuous at $(x, 0)$ for $x \in(0,1)$ if and only if $f(x)=\lim _{w \rightarrow 0^{+}} \varphi(w)$.

(iv) $I_{\varphi, f, g}^{T}$ is continuous at $(1, y)$ for $y \in(0,1)$ if and only if $g(y)=\lim _{w \rightarrow 0^{+}} \varphi(w)$.

(v) $I_{\varphi, f, g}^{T}$ is continuous at $(x, 1)$ and $(0, x)$ for all $x \in[0,1]$ if and only if $\lim _{w \rightarrow+\infty} \varphi(w)=1$.

(vi) $I_{\varphi, f, g}^{T}$ is continuous at $\left(x_{0}, y_{0}\right)$ with $x_{0}, y_{0} \in(0,1)$ if and only if $\varphi$ is continuous at $\frac{t\left(x_{0}\right)}{t\left(y_{0}\right)}$. In this case, $I_{\varphi, f, g}^{T}$ is also continuous at the following points

$$
\left(x, t^{-1}\left(\frac{t(x) t\left(y_{0}\right)}{t\left(x_{0}\right)}\right)\right), \quad \text { for all } x \in(0,1) .
$$

Moreover, from (ii) in the previous result it is easy to see that $I_{\varphi, f, g}^{T}$ is never a continuous function.

Corollary 1. Let $I_{\varphi, f, g}^{T}$ be a strict $T$-power invariant implication. Then at least one of the following conditions holds:

(i) $I_{\varphi, f, g}^{T}$ is discontinuous at $(1,0)$.

(ii) $I_{\varphi, f, g}^{T}$ is discontinuous at $(0,0)$ or $(1,1)$. 
Example 1. Let us show two examples of strict $T$-power invariant implications.

(i) Let us consider $t(s)=\frac{1-s}{s}$ for all $s \in[0,1], f(x)=\frac{1-x}{3}$ for all $x \in(0,1)$, $g(y)=\frac{y}{3}$ for all $y \in(0,1)$ and

$$
\varphi(w)= \begin{cases}0 & \text { if } w=0, \\ \frac{w+1}{w+3} & \text { otherwise }\end{cases}
$$

The corresponding strict $T$-power invariant implication is given by

$$
I_{1}(x, y)= \begin{cases}1 & \text { if }(x, y) \in\{(1,1),(0,0)\}, \\ \frac{1-x}{3} & \text { if } x \in(0,1] \text { and } y=0, \\ \frac{y}{3} & \text { if } x=1 \text { and } y \in(0,1), \\ \frac{y-2 x y+x}{y-4 x y+3 x} & \text { otherwise. }\end{cases}
$$

(ii) Let us consider $t(s)=\frac{1-s}{s}$ for all $s \in[0,1], f(x)=g(y)=0$ for all $x, y \in$ $(0,1)$ and

$$
\varphi(w)=\left\{\begin{array}{l}
w \text { if } w<1, \\
1 \text { otherwise }
\end{array}\right.
$$

The corresponding strict $T$-power invariant implication is given by

$$
I_{2}(x, y)= \begin{cases}0 & \text { if }(x \in(0,1] \text { and } y=0) \text { or }(x=1 \text { and } y \in(0,1)) \\ \frac{(1-x) y}{(1-y) x} & \text { if } 0<x<y<1 \\ 1 & \text { otherwise }\end{cases}
$$

These two fuzzy implication functions are displayed in Fig. 2.

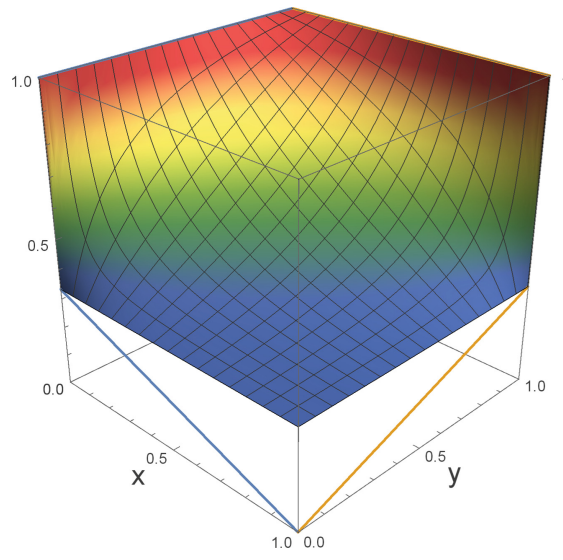

(i) $I_{1}$

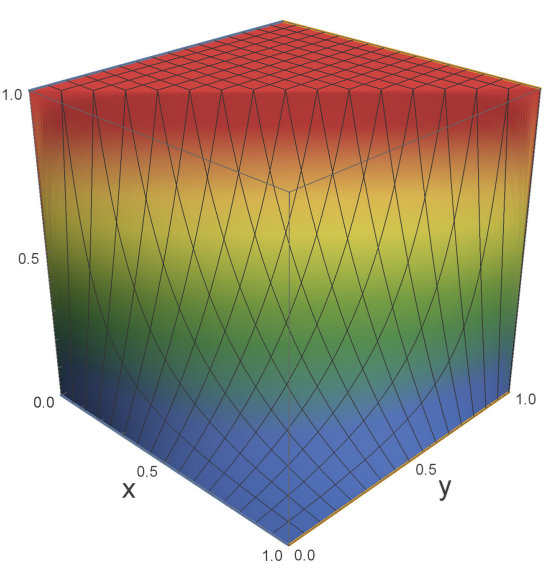

(ii) $I_{2}$

Fig. 2. Plots of fuzzy implication functions given in Example 1. 


\section{Additional Properties}

In [8] it was shown that $T$-power based implications do not satisfy most of the main additional properties of fuzzy implication functions such as the left neutrality principle, the exchange property or the law of importation with respect to any t-norm. This situation represents a problem if we want to consider fuzzy implication functions that are $T$-power invariant but also satisfy other common additional properties. In this section, we generalize the study made in [8] by considering strict $T$-power invariant implications and we show that, in this case, there are choices for $\varphi, f$ and $g$ which ensure that $I_{\varphi, f, g}^{T}$ can also satisfy the properties considered.

First of all, we consider the identity principle and the ordering property. These two properties were already studied in [9] to show that although $I_{\varphi, f, g}^{T}(x, x)$ is constant for all $x \in(0,1),(\mathbf{I P})$ is not guaranteed.

Proposition 3 ([9, Theorem 9]). Let $I_{\varphi, f, g}^{T}$ be a strict T-power invariant implication. Then $I_{\varphi, f, g}^{T}$ satisfies (IP) if and only if $\varphi(1)=1$. In this case, $I_{\varphi, f, g}^{T}$ satisfies $(\mathbf{O P})$ if and only if $\varphi(w)<1$ for all $w<1$.

Next, we show that if a strict $T$-power invariant implication satisfies the left neutrality principle then it is constant to 1 for all $x, y \in(0,1)$.

Proposition 4. Let $I_{\varphi, f, g}^{T}$ be a strict T-power invariant implication. Then $I_{\varphi, f, g}^{T}$ satisfies (NP) if and only if $g(y)=y$ for all $y \in(0,1)$. Moreover, in this case $I_{\varphi, f, g}^{T}$ is given by

$$
I_{\varphi, f, g}^{T}(x, y)= \begin{cases}f(x) & \text { if } x \in(0,1) \text { and } y=0 \\ y & \text { if } x=1 \text { and } y \in[0,1) \\ 1 & \text { otherwise. }\end{cases}
$$

In Fig. 3 we can see the structure of strict $T$-power invariant implications that fulfill (NP) and (OP).
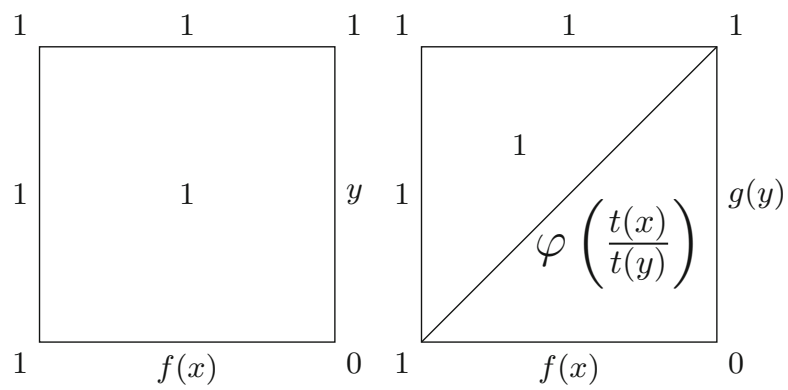

Fig. 3. Structure of strict $T$-power invariant implications that satisfy $(\mathbf{N P})$ and $(\mathbf{O P})$, respectively. 
Now, let us consider the exchange principle. The next result shows that there are five possible configurations of strict $T$-power invariant implications that result in functions that satisfy $(\mathbf{E P})$.

Proposition 5. Let $I_{\varphi, f, g}^{T}$ be a strict $T$-power invariant implication. Then $I_{\varphi, f, g}^{T}$ satisfies (EP) if and only if one of the following conditions hold:

(i) Let $C \in(0,+\infty)$, then $\varphi(w)=t^{-1}\left(\frac{C}{w}\right)$ for all $w \in(0,+\infty)$ and $f(x)=$ $g(y)=0$ for all $x, y \in(0,1)$.

(ii) Let $k \in[0,1]$, then $\varphi(w)=k$ for all $w \in(0,+\infty)$ and $f(x)=g(y)=0$ for all $x, y \in(0,1)$.

(iii) Let $k \in(0,1]$, then $\varphi(w)=k$ for all $w \in(0,+\infty)$ and one of the following conditions holds:

(a) $f(x)=\left\{\begin{array}{l}k \text { if } x \in A, \\ 0 \text { if } x \in(0,1) \backslash A,\end{array}\right.$ where $A$ is $(0, a]$ or $(0, a)$ with $a \in(0,1)$ or $A=\emptyset$ and $\operatorname{Im} g \subseteq(0, k]$.

(b) $f(x)=k$ for all $x \in(0,1)$ and $\operatorname{Im} g \subseteq[0, k]$.

(c) $\operatorname{Im} f \subseteq(0, k], \operatorname{Im} g \subseteq(0, k]$ and $g(y)=y$ for all $y \in \operatorname{Im} f \backslash\{1\}$. Moreover, if $k<1, g$ must additionally satisfy $g(k)=k$.

In Fig. 4 we summarize the possible configurations of strict $T$-power invariant implications that fulfill (EP). Notice that only configuration (i) corresponds to a fuzzy implication function that is not constant in $(0,1)^{2}$.

Example 2. Let us consider $t(s)=\frac{1-s}{s}$ for all $s \in[0,1]$. The corresponding strict $T$-power invariant implication that satisfies $(\mathbf{E P})$ and is non-constant in $(0,1)^{2}$ is given by

$$
I_{3}(x, y)= \begin{cases}1 & \text { if } x=0 \text { and } y=1 \\ 0 & \text { if }(y=0 \text { and } x \in(0,1)) \text { or }(x=1 \text { and } y \in[0,1)) \\ \frac{(1-x) y}{C x-C x y+y-x y} & \text { otherwise }\end{cases}
$$

where $C \in(0,+\infty)$. Note that $I_{3}$ corresponds to the solution given in Proposition 5-(i). In Fig. 5 we can see the plots of some members of this family of fuzzy implication functions for $C=1, C=10$ and $C=100$.

Next, we study the law of importation with respect to a t-norm $T^{*}$. The following result establishes the three possible configurations of strict $T$-power invariant implications that satisfy the law of importation with respect to some t-norm.

Proposition 6. Let $I_{\varphi, f, g}^{T}$ be a strict $T$-power invariant implication and $T^{*}$ a t-norm. Then $I_{\varphi, f, g}^{T}$ satisfies $(\mathbf{L I})$ with respect to $T^{*}$ if and only if one of the following conditions hold:

(i) $\varphi(w)=0$ for all $w \in(0,+\infty), f(x)=g(y)=0$ for all $x, y \in(0,1)$ and $T^{*}$ is a positive t-norm.

(ii) Let $k \in(0,1]$, then $\varphi(w)=k$ for all $w \in(0,+\infty), g(y)=y$ for all $y \in$ $\operatorname{Im} g \backslash\{0,1\}$, and one of the following conditions hold: 


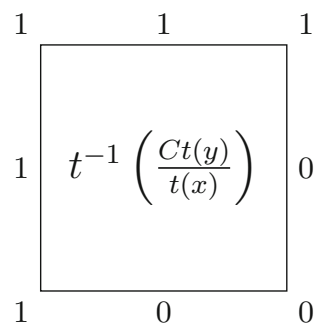

(i) Case (i)

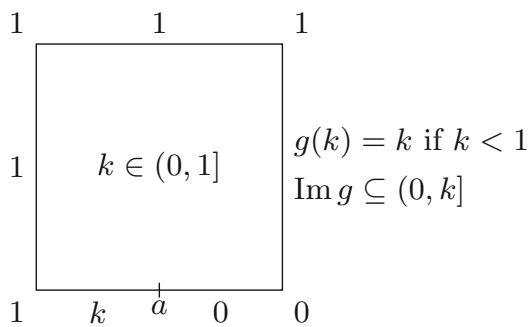

(iii) Case (iii)-(a)

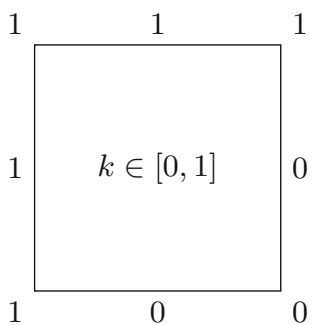

(ii) Case (ii)

1

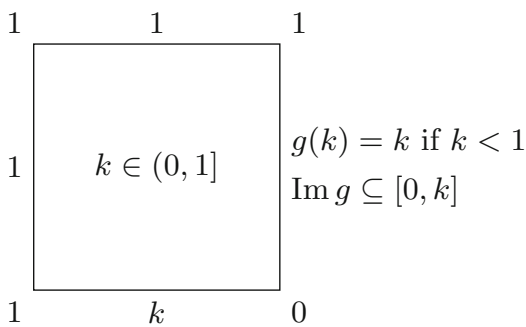

(iv) Case (iii)-(b)

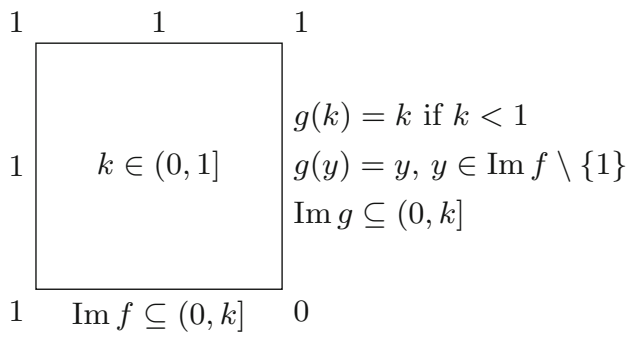

(v) Case (iii)-(c)

Fig. 4. Structure of strict $T$-power invariant implications that satisfy $(\mathbf{E P})$ defined in Proposition 5.

(a) $f(x)=\left\{\begin{array}{l}k \text { if } x \in A, \\ 0 \text { if } x \in(0,1) \backslash A,\end{array}\right.$ where $A$ is $(0, a]$ or $(0, a)$ with $a \in(0,1)$ or $A=\emptyset, T^{*}$ satisfies the following property:

$$
T^{*}(x, y) \in(0,1] \backslash A \text { if and only if } x, y \in(0,1] \backslash A \text {, }
$$

and $\operatorname{Im} g \subseteq(0, k]$.

(b) $0 \notin \operatorname{Im} f, f(x)=k$ for all $\left.x \in \operatorname{Im} T^{*}\right|_{(0,1)^{2}} \backslash\{0\}, g(y)=y$ for all $y \in$ $\operatorname{Im} f \backslash\{1\}$ and $\operatorname{Im} g \subseteq[0, k]$ but $g(y)>0$ for all $y \in(0,1)$ when $f$ is not a function constant to $k$.

Moreover, if $k<1, g$ must additionally satisfy $g(k)=k$ and $T^{*}$ must be a positive t-norm. 


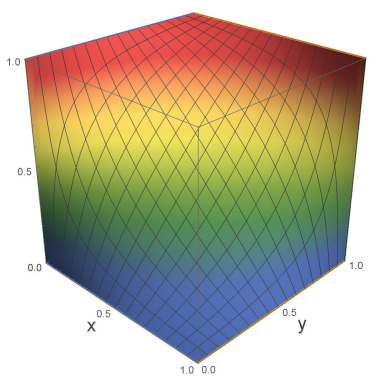

(i) $C=1$

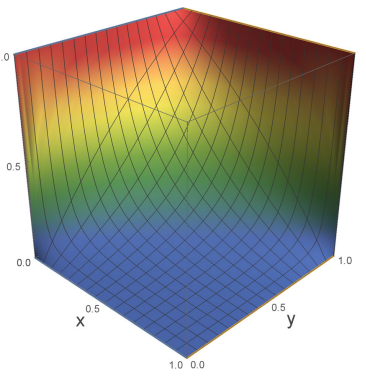

(ii) $C=10$

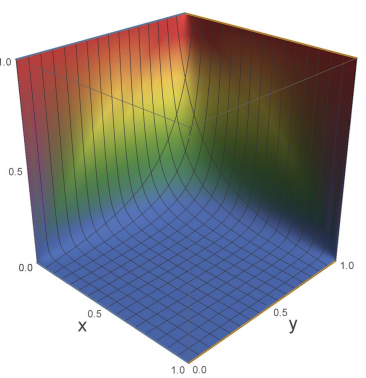

(iii) $C=100$

Fig. 5. Plots of fuzzy implication function given in Example 2 for $C=1, C=10$ and $C=100$.

Example 3. Let us consider $\varphi(w)=\frac{1}{2}$ for all $w \in(0,1), f(x)=\frac{1}{2}$ for all $x \in(0,1)$ and

$$
g(y)=\left\{\begin{array}{l}
y \text { if } y \in\left(0, \frac{1}{4}\right), \\
\frac{1}{2} \text { if } y \in\left[\frac{1}{4}, \frac{1}{2}\right] \\
1 \text { if } y \in\left(\frac{1}{2}, 1\right) .
\end{array}\right.
$$

The corresponding strict $T$-power invariant implication

$$
I_{\varphi, f, g}^{T}(x, y)=\left\{\begin{array}{l}
y \text { if } x=1 \text { and } y \in\left[0, \frac{1}{4}\right) \\
1 \text { if } x=0 \text { or } y=1 \text { or }\left(x=1 \text { and } y \in\left(\frac{1}{2}, 1\right)\right) \\
\frac{1}{2} \text { otherwise }
\end{array}\right.
$$

satisfies the law of importation with respect to any positive t-norm, for instance the minimum t-norm $T_{M}(x, y)=\min (x, y)$ or the product t-norm $T_{P}(x, y)=x y$.

Remark 1. Let us consider a strict $T$-power invariant implication under the conditions of (iii)-(a) in Proposition 6 where $A=(0, a)$ with $a \in(0,1)$ and $k \in(0,1)$. Then, this fuzzy implication function satisfies the law of importation with respect to any positive t-norm $T^{*}$ such that $T^{*}(x, y) \in[a, 1]$ if and only if $x, y \in[a, 1]$. For instance, $T^{*}$ can be a continuous positive t-norm with $a$ as an idempotent element. However, a further study must be made in order to characterize all t-norms that fulfill such condition.

Finally, we analyze under which conditions strict $T$-power invariant implications satisfy the iterative boolean law. In this case, we see that $\varphi$ needs to be a constant function or its only possible values are 0 and 1.

Proposition 7. Let $I_{\varphi, f, g}^{T}$ be a strict $T$-power invariant implication. Then $I_{\varphi, f, g}^{T}$ satisfies (IB) if and only if one of the following conditions hold:

(i) $\operatorname{Im} \varphi \subseteq\{0,1\}, \varphi$ is not constant to 1 and $f(x)=g(y)=0$ for all $x, y \in(0,1)$.

(ii) Let $k \in(0,1]$, then $\varphi(w)=k$ for all $w \in(0,+\infty), \operatorname{Im} f \subseteq\{0, k\}, \operatorname{Im} g \subseteq$ $[0, k]$ and $g(y)=y$ for all $y \in \operatorname{Im} g \backslash\{0,1\}$. 
Example 4. Let $T$ be any strict t-norm, $t$ an additive generator of $T, f(x)=$ $g(y)=0$ for all $x, y \in(0,1)$ and

$$
\varphi(w)=\left\{\begin{array}{l}
0 \text { if } w<\frac{1}{a}, \\
1 \text { otherwise }
\end{array}\right.
$$

with $a \in(0,+\infty)$. Then, the corresponding strict $T$-power invariant implication

$$
I_{\varphi, f, g}^{T}(x, y)=\left\{\begin{array}{l}
0 \text { if } y<t^{-1}(a t(x)), \\
1 \text { otherwise, }
\end{array}\right.
$$

satisfies the iterative boolean law.

Remark 2. According to Propositions 4, 5, 6 and 7 all strict $T$-power invariant implications that satisfy $(\mathbf{N P}),(\mathbf{E P}),(\mathbf{I B})$ or $(\mathbf{L I})_{\mathbf{T}}$ except case (i) in Proposition 5 and case (i) in Proposition 7 , are given by a $\varphi$ which is constant in $(0,+\infty)$. Therefore, these fuzzy implication functions are given by an expression which is independent from the generator of the corresponding t-norm. Then, they are also $T$-power invariant with respect to any strict t-norm. This fact reflects that imposing the $T$-power invariance with some additional property is very restrictive and usually results in degenerated solutions.

\section{Intersection with the Main Families of Fuzzy Implication Functions}

It is well-known that an important step when studying a new family of fuzzy implication functions is to study their intersection among other families of fuzzy implication functions. In this section we investigate whether the family of strict $T$-power invariant implications intersects with five of the most well-known families of fuzzy implication functions. Let us denote the following families of fuzzy implication functions:

$$
\begin{aligned}
\mathbb{I}_{\varphi, f, g}^{T} & - \text { the family of all strict } T \text {-power invariant implications; } \\
\mathbb{I}_{\mathbb{S}, \mathbb{N}} & - \text { the family of all }(S, N) \text {-implications; } \\
\mathbb{I}_{\mathbb{T}} & - \text { the family of all } R \text {-implications; } \\
\mathbb{I}_{\mathbb{Q} \mathbb{L}} & - \text { the family of all } Q L \text {-implications; } \\
\mathbb{I}_{\mathbb{F}} & - \text { the family of all } f \text {-generated implications; } \\
\mathbb{I}_{\mathbb{G}} & - \text { the family of all } g \text {-generated implications. }
\end{aligned}
$$

In [8] it was pointed out that $T$-power based implications have no intersection with all the above families because they do not satisfy the left neutrality principle. However, we have seen that there are choices for $f, g$ and $\varphi$ such that the corresponding strict $T$-power invariant implication satisfies (NP). For instance, the following fuzzy implication function satisfies both (NP) and (EP):

$$
I^{*}(x, y)= \begin{cases}f(x) & \text { if } x \in(0,1) \text { and } y=0 \\ y & \text { if } x=1 \text { and } y \in[0,1) \\ 1 & \text { otherwise }\end{cases}
$$


where $f:(0,1) \rightarrow[0,1]$ is any decreasing function with $\operatorname{Im} f \subseteq(0,1]$. Notice that if we choose $f(x)=1$ for all $x \in(0,1)$ we obtain the well-known Weber implication

$$
I_{\mathbf{W B}}(x, y)=\left\{\begin{array}{l}
y \text { if } x=1 \text { and } y \in[0,1] \\
1 \text { otherwise. }
\end{array}\right.
$$

Thanks to the study of the previous section, we are able to prove that strict $T$-power invariant implications have non-empty intersection with $R, Q L$ and $(S, N)$-implications. Indeed, the following proposition provides the complete characterization of the intersections of interest.

Proposition 8. The following equalities are true:

$-\mathbb{I}_{\varphi, f, g}^{T} \cap \mathbb{I}_{\mathbb{T}}=I_{W B}$.

$-\mathbb{I}_{\varphi, f, g}^{T} \cap \mathbb{I}_{\mathbb{S}, \mathbb{N}}=\mathbb{I}_{\varphi, f, g}^{T} \cap \mathbb{I}_{\mathbb{Q L}}=I^{*}$.

$-\mathbb{I}_{\varphi, f, g}^{T} \cap \mathbb{I}_{\mathbb{F}}=\mathbb{I}_{\varphi, f, g}^{T} \cap \mathbb{I}_{\mathbb{G}}=\emptyset$.

Notice that although the intersection of strict $T$-power invariant implications and $R, Q L$ and $(S, N)$-implications is not empty, the fuzzy implication functions that belong to this intersection are constant to 1 in $(0,1)^{2}$. Therefore, we can conclude that the $T$-power invariance property with respect to a strict t-norm is not satisfied for almost all fuzzy implication functions that belong to the most well-known families of fuzzy implication functions. In this sense, it is relevant to study strict $T$-power invariant implications as a new family of fuzzy implication functions.

\section{Conclusions and Future Work}

In this paper, the most usual additional properties of fuzzy implication functions have been studied for the family of strict $T$-power invariant implications, those fuzzy implication functions which are invariant with respect to powers of a strict t-norm. The results show that members of this family can satisfy other additional properties in addition to the invariance property. While for some properties such as $(\mathbf{N P})$ or $(\mathbf{L I})_{\mathbf{T}}$, the only solutions are fuzzy implication functions which are constant in $(0,1)^{2}$, other properties such as $(\mathbf{I B}),(\mathbf{O P})$ or $(\mathbf{E P})$ provide richer non-constant solutions. Note that these results are in fact characterizations of all fuzzy implication functions which satisfy both the invariance with respect to powers of a strict t-norm and the corresponding other additional property. Finally, from these results, the intersections with the most well-known families is fully determined leading to some $R, Q L$ and $(S, N)$-implications which are also invariant with respect to powers of a strict t-norm.

As future work, we want to perform a similar study for the family of nilpotent $T$-power invariant implications and also for those fuzzy implication functions which are invariant with respect to powers of an ordinal sum t-norm.

Acknowledgment. This paper has been partially supported by the Spanish Grant TIN2016-75404-P, AEI/FEDER, UE. Raquel Fernandez-Peralta benefits from the fellowship FPU18/05664 granted by the Spanish Ministry of Science, Innovation and Universities within the Training university lecturers (FPU) program. 


\section{References}

1. Baczyński, M., Jayaram, B.: Fuzzy Implications. Studies in Fuzziness and Soft Computing, vol. 231. Springer, Heidelberg (2008). https://doi.org/10.1007/978-3540-69082-5

2. Baczynski, M., Jayaram, B., Massanet, S., Torrens, J.: Fuzzy implications: past, present, and future. In: Kacprzyk, J., Pedrycz, W. (eds.) Springer Handbook of Computational Intelligence, pp. 183-202. Springer, Heidelberg (2015). https://doi. org/10.1007/978-3-662-43505-2_12

3. Fodor, J.C., Roubens, M.: Fuzzy Preference Modelling and Multicriteria Decision Support. Kluwer Academic Publishers, Dordrecht (1994)

4. Klement, E., Mesiar, R., Pap, E.: Triangular Norms. Kluwer Academic Publishers, Dordrecht (2000)

5. Mas, M., Monserrat, M., Torrens, J., Trillas, E.: A survey on fuzzy implication functions. IEEE Trans. Fuzzy Syst. 15(6), 1107-1121 (2007)

6. Massanet, S.: Tidying up the mess of classes of fuzzy implication functions. In: Halas, R., Gagolewski, M., Mesiar, R. (eds.) Book of Abstracts of the 10th International Summer School on Aggregation Operators, pp. 1-2. Olomouc University (2019)

7. Massanet, S., Recasens, J., Torrens, J.: Corrigendum to "Fuzzy implication functions based on powers of continuous t-norms" [Int. J. Approx. Reason. 83, 265-279 (2017)]. Int. J. Approx. Reason. 104, 144-147 (2019)

8. Massanet, S., Recasens, J., Torrens, J.: Fuzzy implication functions based on powers of continuous t-norms. Int. J. Approx. Reason. 83, 265-279 (2017)

9. Massanet, S., Recasens, J., Torrens, J.: Some characterizations of T-power based implications. Fuzzy Sets Syst. 359, 42-62 (2019)

10. Mizumoto, M., Zimmermann, H.J.: Comparison of fuzzy reasoning methods. Fuzzy Sets Syst. 8, 253-283 (1982)

11. Pradera, A., Beliakov, G., Bustince, H., De Baets, B.: A review of the relationships between implication, negation and aggregation functions from the point of view of material implication. Inf. Sci. 329, 357-380 (2016)

12. Walker, C.L., Walker, E.A.: Powers of t-norms. Fuzzy Sets Syst. 129(1), 1-18 (2002) 